\title{
Correction of Channel Imbalance for MIMO SAR Using Stepped-Frequency Chirps
}

\author{
Xiulian Luo, ${ }^{1,2}$ Yunkai Deng, ${ }^{1}$ Robert Wang, ${ }^{1}$ Lei Guo, ${ }^{1,2}$ and Mingjiang Wang ${ }^{1,2}$ \\ ${ }^{1}$ Institute of Electronics, Chinese Academy of Sciences, Beijing 100190, China \\ ${ }^{2}$ Graduate University of the Chinese Academy of Sciences, Beijing 100080, China \\ Correspondence should be addressed to Xiulian Luo; xiaoluo6070@126.com
}

Received 20 December 2013; Accepted 19 February 2014; Published 3 April 2014

Academic Editor: Wei Xu

Copyright (C) 2014 Xiulian Luo et al. This is an open access article distributed under the Creative Commons Attribution License, which permits unrestricted use, distribution, and reproduction in any medium, provided the original work is properly cited.

\begin{abstract}
To simultaneously achieve two-dimensional high resolution and wide swath in synthetic aperture radar (SAR), azimuth MIMO structure combined with stepped-frequency chirp signals was developed via splitting the antenna into $N$ subapertures. During transmitting each subaperture transmits a chirp pulse at a different carrier frequency, while during receiving every subaperture receives the $N$ scattered pulses at the same time. Separating the $N$ scattered pulses received by each subaperture and downlinking them to the ground yield $N^{2}$ different signal paths. Due to the dedicated network in the SAR system, the channel imbalance is inevitable. To correct the channel imbalance, this paper presents an external calibration method, where the channel characteristics are estimated from the peak value of a strong point target for each channel. Simulation and real raw data experiments are performed to validate the proposed method.
\end{abstract}

\section{Introduction}

Resolution and swath width are two key specifications for the spaceborne stripmap SAR. However, high azimuth resolution and wide range swath imaging pose contradicting requirement on conventional SAR system design. In order to overcome this inherent contradiction, the displaced phase center antenna (DPCA) techniques [1-3] and the multipleinput and multiple-output (MIMO) antenna techniques [47] are introduced to acquire additional spatial sampled information. On the other hand, the range resolution, determined by the pulse bandwidth, has to be increased to match the azimuth resolution. However, transmitting and receiving wideband signals burden the active electronically steered array (AESA) antenna and the echo sampled equipment $[8,9]$, thus confining the range resolution. Fortunately, a wideband chirp signal can be synthesized by a group of narrowband subchirps centered at stepped frequencies, which requires the synthetic bandwidth technique [8-10].

As the synthetic bandwidth technique will reduce the number of equivalent phase centers (EPCs), the additional sampled information in DPCA SAR is actually used to increase the range bandwidth other than the equivalent PRF [11]. Therefore, this paper focuses on the MIMO SAR using stepped-frequency chirps (STFC) $[4,5]$, where both the equivalent PRF and the range bandwidth can be $N$ times increased. However, as this MIMO system yields $N^{2}$ different signal paths, the channel imbalance is inevitable. For each subband, the collected data is from a DPCA system, where the channel imbalance can be regarded as the inner-band imbalance which results in azimuth ambiguities [12]. The imbalance among different subbands can be regarded as the interband imbalance which leads to degradation of the range performance after synthetic bandwidth [13].

Therefore, in order to acquire high quality images, effective correction of the channel imbalance is indispensable. The available correction methods can be classified as two categories: methods on internal calibration [14] and methods on raw data $[15,16]$. However, all these correction methods only either handled the inner-band imbalance in the DPCA system with single subband $[15,16]$ or concerned the multiple subbands system with single subaperture [14]. In [14], Deng et al. gave the internal calibration approach, where the imbalance information is obtained from the internal calibration 
data acquired from the calibration subsystem. This method can be extended to the MIMO system using STFC. Nevertheless, $N^{2}$ internal calibration subsystems are required, which dramatically increases the complexity of the SAR systems. In addition, it cannot correct imbalance introduced by the antennas. The subspace projection method proposed in [16] and the azimuth cross-correlation method presented in [15] do not require additional subsystem. However, they cannot correct the interband imbalance, as the phase difference caused by different carrier frequencies is range dependent and hard to compensate.

Usually, the channel imbalance information (phase and amplitude) does not change within a certain illumination time [15]. Moreover, many artificial corner reflectors have been distributed here and there for radiometric calibration [17]. Therefore, this paper proposes an external calibration method where the channel imbalance information is estimated from the peak value of a corner reflector or a strong point target in $N^{2}$ small complex images. Afterwards, the estimated channel errors are used to correct the channel imbalance when imaging for much larger data.

Succeeding sections are organized as follows. In Section 2, the MIMO SAR using STFC is conceptually designed and the corresponding image formation processing is briefly summarized. In Section 3, the external correction method is presented, followed by the simulation and real raw data experiments in Section 4 to validate the proposed method. Finally, the conclusion is drawn in Section 5.

\section{MIMO SAR Using Stepped-Frequency Chirps}

2.1. Conceptual Design. The MIMO SAR simultaneously transmits a set of stepped-frequency chirps, regarded as subbands, via multiple subapertures. During receiving, every subaperture receives all the $N$ subbands in one PRI. The schematic diagram and the corresponding timeline of transmitted pulses and received echoes are illustrated in Figure 1, where the circles represent the location of the equivalent phase centers (EPCs) of the subbands, and the number inside the circles represents the sequence number of the subbands.

In order to separate two frequency-adjacent subbands and absent gaps from the connection of subbands at the same time, the subband bandwidth $B_{s}$ is required to be equal to the frequency step $\Delta f$. The relationship of the time frequency for the transmitted pulses is illustrated in Figure 2, where $f_{m}$ $(m=1,2, \ldots, N)$ is the carrier frequency of the $m$ th subband.

The baseband echo of the $m$ th subband for each subaperture is acquired as follows. Demodulate the radio frequency (RF) pulses with the carrier frequency $f_{m}$. Then filter the demodulated signal with an analog low pass filter (LPF) whose bandwidth is greater than $B_{s}$. Afterwards, convert the analog signal to a digital signal via an $\mathrm{AD}$ convertor with a sampling rate greater than the cutoff frequency of the LPF. As illustrated in Figure 3, due to the imperfect filtering and the Gibbs phenomenon of the signal spectrum, some spectral component of one subband is leaked into the adjacent subband, thus resulting in subband interference. In

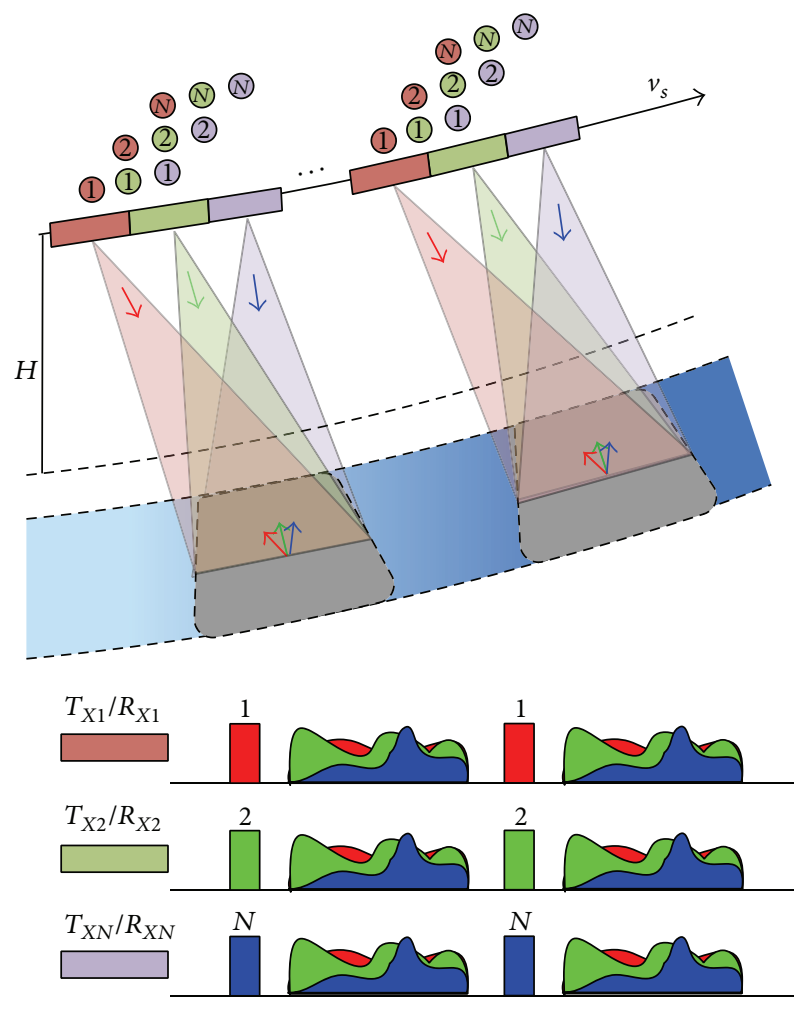

FIGURE 1: Schematic diagram of the MIMO SAR using STFC.

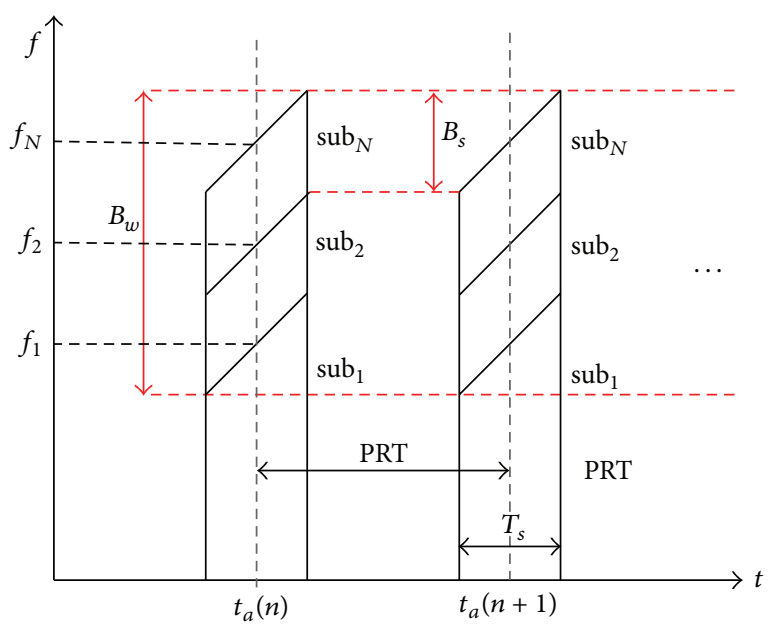

FIGURE 2: Relationship of time frequency of transmitted pulses.

order to reduce the interference, a digital rectangular window can be applied to the sampled data before imaging. Then, the interference only results from the spectral component outside the spectrum bandwidth, as illustrated by the blueshaded region in Figure 3. As stated in [13], the influence of the subband interference under this situation can be ignored because it results in $-47 \mathrm{~dB}$ ambiguities in range. In fact, when the subband bandwidth is larger, the ambiguities in range will be reduced further. This is because the relative amplitude of the ambiguities is determined by relating the leakage energy 


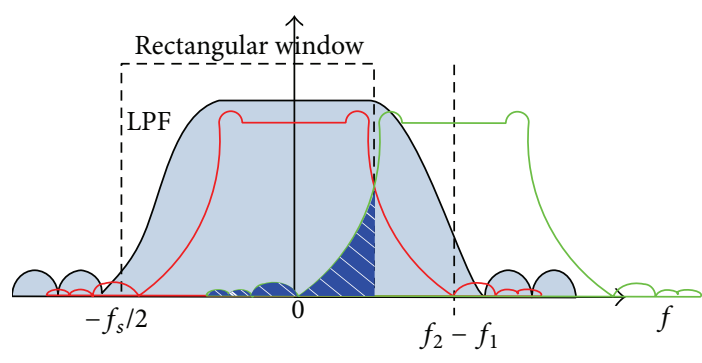

FIgURE 3: Separation of two frequency-adjacent subbands.

to the whole energy [13]. Moreover, the leakage energy is inversely proportional to the bandwidth-time product.

Therefore, in this paper, for each subaperture, adjacent subbands are assumed to be completely separated; that is, the subband interference is not considered.

2.2. Image Formation Processing. When the uniform sampling condition

$$
\frac{1}{\mathrm{PRF}}=\frac{l_{a z}}{2 v_{s}} \cdot N
$$

with PRF the system pulse repetition frequency, $l_{a z}$ the length of the subaperture, and $v_{s}$ the velocity of the sensor, is not satisfied, multichannel reconstruction [1] is required. The reference position during the multichannel reconstruction should be the center of the whole antenna to make the EPCs to be synthesized coincide spatially. Afterwards, the frequency domain bandwidth synthesis method $[9,10]$ is applied to acquire a large bandwidth pulse, followed by the conventional imaging. It should be noted that the synthesized signal should be firstly converted to the original echo domain if the CS imaging algorithm is adopted. The block diagram of the image formation processing for this MIMO SAR system is presented in Figure 4. Assume that $N=2$, and then the variation of the distribution of EPCs introduced by multichannel reconstruction and bandwidth synthesis is shown in Figure 5 (ignore the first row and the dashed circles, which will be used in Section 4).

\section{Correction of the Channel Imbalance}

In this section, the correction of both amplitude and phase imbalance among channels will be presented. As this correction method is based on a focused strong point target in $N^{2}$ small complex images, separating imaging for each subband and each subaperture is required.

The base-band echo for the $m$ th subband (transmitted by the $m$ th subaperture) received by the $n$th subaperture is

$$
\begin{aligned}
S_{m, n}(\tau, \eta)= & N_{m, n}(\tau, \eta)+A_{m, n} \\
& \cdot \exp \left(j \varphi_{m, n}\right) \cdot \operatorname{rect}\left(\frac{\tau-R_{m, n}(\eta) / c}{T_{r}}\right)
\end{aligned}
$$

$$
\begin{aligned}
& \times \exp \left\{-j 2 \pi f_{m} \cdot\left(\frac{R_{m, n}(\eta)}{c}\right)\right\} \\
& \cdot \exp \left\{j \pi k_{r}\left(\tau-\frac{R_{m, n}(\eta)}{c}\right)^{2}\right\},
\end{aligned}
$$

with $\tau$ the range time, $\eta$ the azimuth time, $k_{r}$ the chirp rate, $c$ the light velocity, $T_{r}$ the pulse duration, $A_{m, n}$ the amplitude characteristic, $\varphi_{m, n}$ the phase characteristic, $N_{m, n}(\tau, \eta)$ the system noise and the echo of other targets, and $R_{m, n}(\eta)$ the instantaneous distance in propagation between the transmitter and the receiver for the corner reflector (point target). Applying the Taylor series expansion and ignoring the quadratic phase termquadratic term error during the equivalence of the two-way slant ranges, $R_{m, n}(\eta)$ can be approximated as

$$
R_{m, n}(\eta)=R\left(\eta-\Delta \eta_{m, n}\right)
$$

where

$$
\Delta \eta_{m, n}=\frac{(m+n-N-1) l_{a z}}{2 v_{s}},
$$

is the azimuth time interval between the corresponding EPC and the center of the whole antenna, and

$$
R(\eta)=2 \sqrt{R_{0}^{2}+\left(v_{s} \eta-x_{0}\right)^{2}}
$$

represents the two-way slant range from the center of the whole antenna to the point target. In (5), $R_{0}$ and $x_{0}$ indicate the slant range and azimuth coordinates of the point target, respectively.

First, the imaging for the $N^{2}$ echoes is independently performed. Due to lower PRF than Nyquist sampling rate, strong azimuth ambiguities of the point target appear in the $N^{2}$ images. However, the ambiguities are far away from the real point target, thus scarcely impacting the estimation. The imaging result can be approximated as [18]

$$
\begin{aligned}
S_{m \cdot n}(\tau, \eta)= & N_{m, n}^{\prime}(\tau, \eta)+N_{m, n, \mathrm{amb}}^{\prime}(\tau, \eta) \\
& +A_{k} \cdot \exp \left(j \varphi_{k}\right) \cdot G \cdot p_{r}\left(\tau-\frac{2 R_{0}}{c}\right) \\
& \cdot \exp \left\{-j 2 \pi f_{m} \cdot\left(\frac{2 R_{0}}{c}\right)\right\} \cdot p_{a, m, n}\left(\eta-\frac{x_{0}}{v_{s}}\right) \\
& +A_{k} \cdot \exp \left(j \varphi_{k}\right) \cdot p_{r}\left(\tau-\frac{2 R_{0}}{c}\right)
\end{aligned}
$$




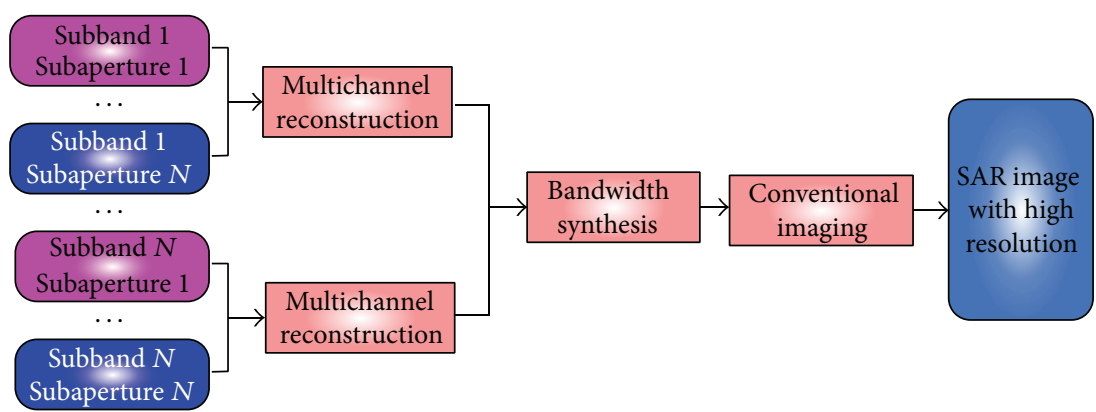

FIGURE 4: Block diagram of the image formation processing.

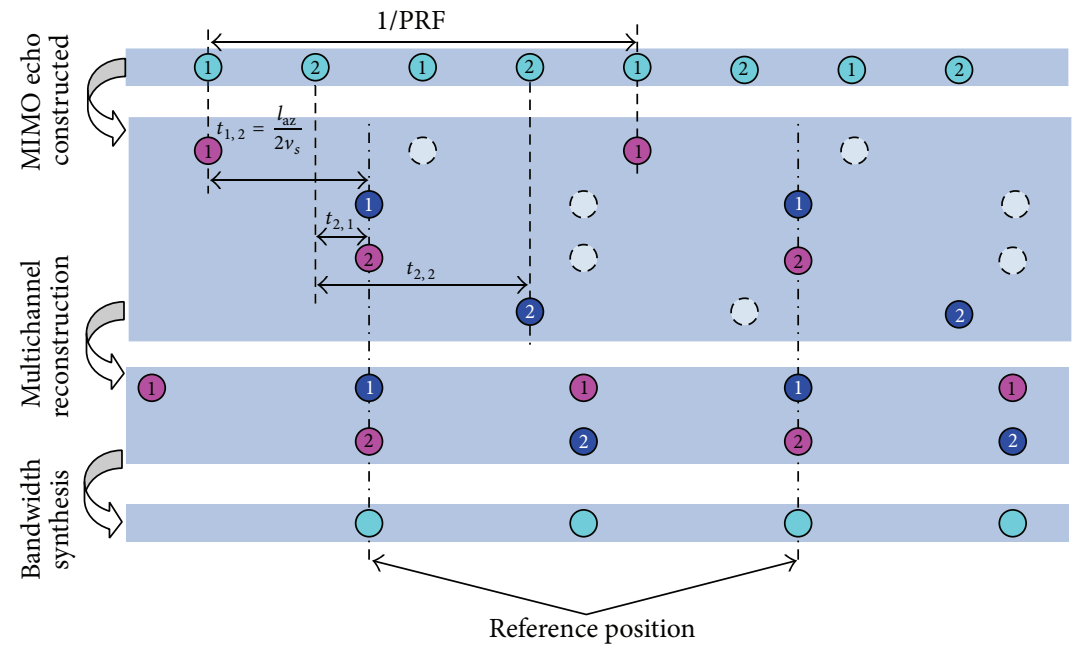

FIgURE 5: Distribution of the EPCs.

$$
\begin{aligned}
\cdot \exp \left\{-j 2 \pi f_{m} \cdot\left(\frac{2 R_{0}}{c}\right)\right\} \\
\times \sum_{i=-N+1, i \neq 0}^{N-1} G_{\mathrm{amb}, i} \\
\cdot p_{a, m, n}\left(\eta-\frac{x_{0}}{v_{s}}+i \cdot \frac{\mathrm{PRF}}{k_{a}}\right),
\end{aligned}
$$

where $k_{a}$ is the azimuth chirp rate, $G$ and $G_{\mathrm{amb}, i}$ are the gain resulting from the imaging of the "real" part and the ambiguous part of the point target, respectively, $N_{m, n}^{\prime}(\tau, \eta)$ and $N_{m, n, \mathrm{amb}}^{\prime}(\tau, \eta)$ are the "real" and ambiguous response of other targets and noise, and $p_{a, m, n}$ and $p_{r}$ are the amplitude of the azimuth and range impulse responses, two sinc-like functions [18]. According to (3), $p_{a, m, n}(\eta)$ can be rewritten as

$$
p_{a, m, n}(\eta)=p_{a}\left(\eta-\Delta \eta_{m, n}\right)
$$

where $p_{a}$ is also a sinc-like function.

Considering that the peak of the corner reflector is generally much greater than other terms at position $\left(R_{0}, x_{0}\right)$, only the "real" response of the corner reflector is taken into account when deriving the correction method, which is

$$
\begin{aligned}
S_{m, n}(\tau, \eta)= & A_{m, n} \cdot \exp \left(j \varphi_{m, n}\right) \cdot G \cdot p_{r}\left(\tau-\frac{2 R_{0}}{c}\right) \\
& \times \exp \left\{-j 2 \pi f_{m} \cdot\left(\frac{2 R_{0}}{c}\right)\right\} \\
& \cdot p_{a}\left(\eta-\frac{x_{0}}{v_{s}}-\Delta \eta_{m, n}\right) .
\end{aligned}
$$

Second, the peak position and value of the corner reflector are estimated from these $N^{2}$ small complex images. In order to make the estimation more accurate, the $2 \mathrm{D}$-interpolation over the peak point and its surrounding points is performed. Consequently, the peak position of the corner reflector is acquired to be $\bar{\tau}_{m, n} \approx 2 R_{0} / c$. The amplitude of the peak is $\bar{A}_{m, n} \approx A_{m, n}$, and the phase of the peak is

$$
\bar{\phi}_{b, m, n} \approx \varphi_{m, n}-2 \pi f_{m} \cdot \bar{\tau}_{m, n} .
$$

In fact, in order to reduce the influence of the noise, $\overline{A_{k}}$ and $\bar{\phi}_{b, k}$ are obtained by averaging the values of the points within the two-dimensional $1 \mathrm{~dB}$ main lobe. The first channel 
(subband 1 and subaperture 1) is set as the reference channel. Then the estimated amplitude error matrix is

$$
\overline{\mathbf{A}_{\mathbf{E}}}=\operatorname{diag} \frac{\left\{\bar{A}_{1,1}, \bar{A}_{1,2}, \ldots, \bar{A}_{1, N}, \ldots, \bar{A}_{N, 1}, \bar{A}_{N, 2}, \ldots, \bar{A}_{N, N}\right\}}{\bar{A}_{1,1}}
$$

Third, compensate the inherent phase caused by the offset of the carrier frequencies $f_{m}$ from the center frequency of the synthesized wideband signal, $f_{c}$. After this, the phase of the peak value is changed as

$$
\bar{\phi}_{m, n}=\bar{\phi}_{b, m, n}+2 \pi \Delta f_{m} \cdot \bar{\tau}_{m, n} \approx \varphi_{m, n}-2 \pi f_{c} \cdot\left(\frac{2 R_{0}}{c}\right) \text {, }
$$

where

$$
\Delta f_{m}=\left(m-\frac{1+N}{2}\right) \Delta f, \quad m=1, \ldots, N
$$

Therefore, the estimated phase error matrix can be expressed as

$$
\begin{aligned}
& \overline{\Phi_{\mathrm{E}}}=\left(\operatorname { d i a g } \left\{\exp \left(j \bar{\phi}_{1,1}\right), \ldots, \exp \left(j \bar{\phi}_{1, N}\right), \ldots,\right.\right. \\
&\left.\left.\exp \left(j \bar{\phi}_{N, 1}\right), \ldots, \exp \left(j \bar{\phi}_{N, N}\right)\right\}\right) \\
& \times\left(\exp \left(j \bar{\phi}_{1,1}\right)\right)^{-1} .
\end{aligned}
$$

Finally, correct the channel imbalance according to (10) and (13). The correction matrix is

$$
\mathrm{C}=\left(\overline{\mathrm{A}_{\mathrm{E}}} \overline{\Phi_{\mathrm{E}}}\right)^{-1}
$$

Therefore, the correction is implemented as

$$
\begin{array}{r}
\mathbf{S}_{c}=\mathbf{C} \cdot\left[S_{1,1}(\tau, \eta), \ldots, S_{1, N}(\tau, \eta), \ldots,\right. \\
\left.S_{N, 1}(\tau, \eta), \ldots, S_{N, N}(\tau, \eta)\right]^{T} .
\end{array}
$$

It should be noted that the data size of $S_{m, n}(\tau, \eta)$ in (2) can be much smaller than that in (15) to improve the processing efficiency. After correction, the balanced data $S_{c}$ is used to form the final image with high resolution. The whole processing is shown in Figure 6, where the image formation processing strategy is presented in Figure 4.

\section{Experimental Results}

4.1. Simulation Experiment. In the simulation, the 2D imaging of a plane scene containing a letter " $A$ " and a 4-time $(6 \mathrm{~dB})$ brighter point target is performed, and this point target is inside the letter "A." The simulation parameters are specified in Table 1.
TABLE 1: System parameters.

\begin{tabular}{lcc}
\hline Parameter & Symbol & Value \\
\hline Center frequency $(\mathrm{GHz})$ & $f_{c}$ & 9.685 \\
Slant range of scene center $(\mathrm{km})$ & $R_{0}$ & 30 \\
Sensor velocity $(\mathrm{m} / \mathrm{s})$ & $v_{s}$ & 215 \\
Subaperture length $(\mathrm{m})$ & $l_{\mathrm{az}}$ & 2.5 \\
System PRF $(\mathrm{Hz})$ & $\mathrm{PRF}$ & 140 \\
Transmit pulse length (us) & $T_{p}$ & 10 \\
Number of subbands/subapertures & $N$ & 2 \\
Transmitted pulse bandwidth $(\mathrm{MHz})$ & $B_{s}$ & 60 \\
Sampling rate in range $(\mathrm{MHz})$ & $F_{r}$ & 72 \\
SNR (dB) & $\mathrm{SNR}$ & 6 \\
\hline
\end{tabular}

The accurate amplitude and phase errors are given in the 2nd and 3rd columns of Table 2, respectively. The first channel is set as the reference channel. According to the estimation method proposed in the last section, the amplitude and phase errors are estimated and then presented in the 4 th and 5th columns of Table 2, respectively. The imaging results without and with correction of the channel imbalance are shown in Figures $7(\mathrm{a})$ and $7(\mathrm{~b})$, respectively. The range comparison of the point target and its surroundings is shown in Figure $7(\mathrm{c})$. From Figure 7, one can observe that the ambiguities in azimuth (caused by the inner-band imbalance) and the raising of the side-lobes in range (stemming from the interband imbalance) are removed by the correction of channel imbalance.

From the simulation experiment, it is concluded that the proposed correction (compensation) method can correct both the inner-band and the interband imbalance for the simulated MIMO SAR data.

4.2. Real Raw Data Experiment. For the real raw data, the imaging scene is much more complicated than the simulated data, thus possibly affecting the estimation accuracy. As the real raw data collected by a MIMO SAR using STFC is not available, in this subsection, the proposed correction method is assessed with an X-band airborne SAR using a single aperture and two STFCs which are transmitted in two consecutive PRIs. In this SAR, both the subband bandwidth and the frequency step are $30 \mathrm{MHz}$, the original PRF is $1600 \mathrm{~Hz}$, and the sensor velocity is $215 \mathrm{~m} / \mathrm{s}$. The distribution of the original EPCs is illustrated by the first row in Figure 5.

To validate the proposed correction method on the real raw data, the echo data for the MIMO system have to be firstly constructed. As discussed before, in the MIMO system using STFCs, the echo for each subband can be regarded from a DPCA system [1]. Further, in the DPCA system, the data of each channel is equivalent to the counterpart single channel data processed by an azimuth time delay function and then $N$ times subsampling [2]. Herein, the counterpart single channel data is the original subband data, and the delay function in Doppler domain for the $m$ th subband received by the $n$th subaperture is set to

$$
H_{m, n}\left(f_{\eta}\right)=\exp \left\{-j 2 \pi f_{\eta} \cdot t_{m, n}\right\},
$$




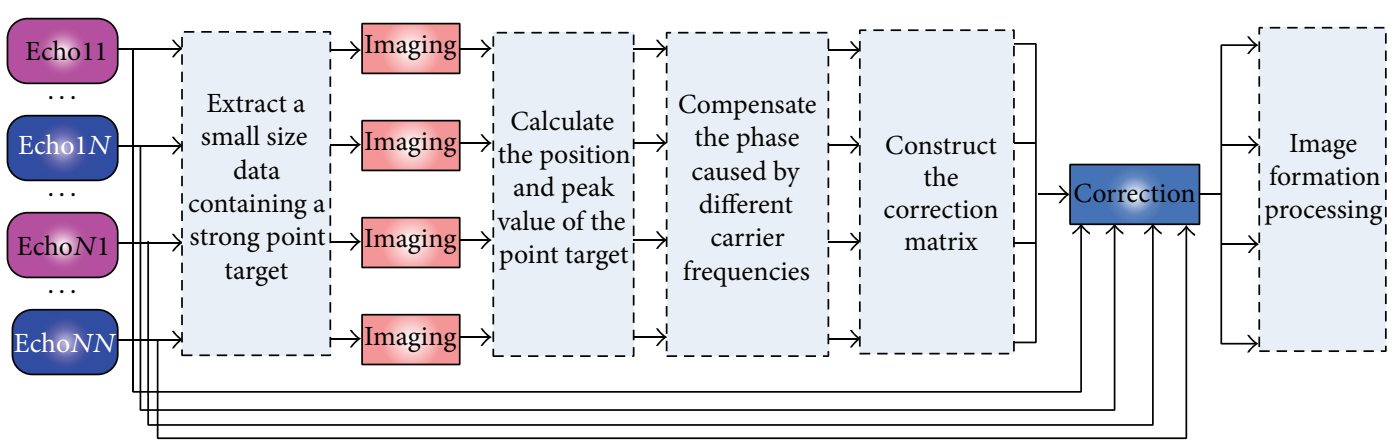

FIGURE 6: Block diagram of the channel imbalance correction.

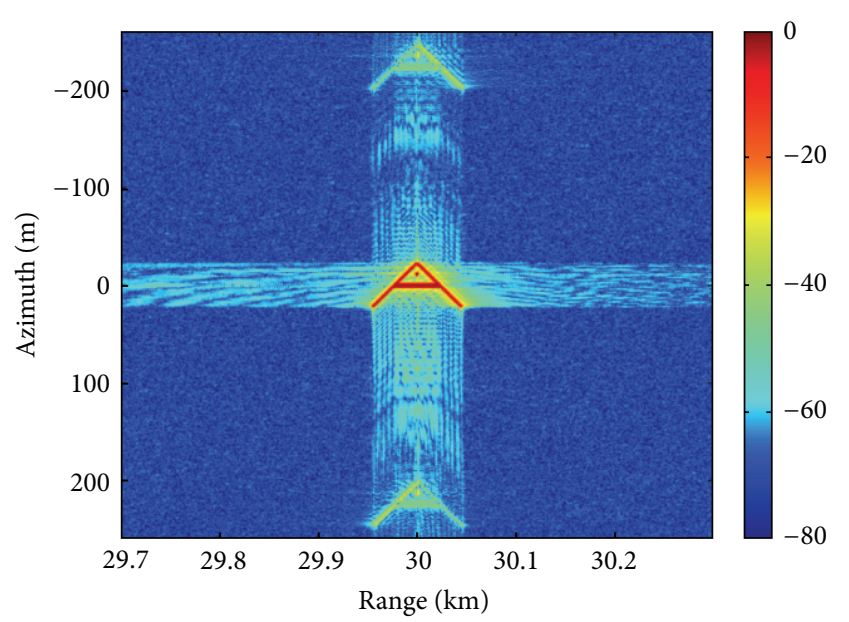

(a)

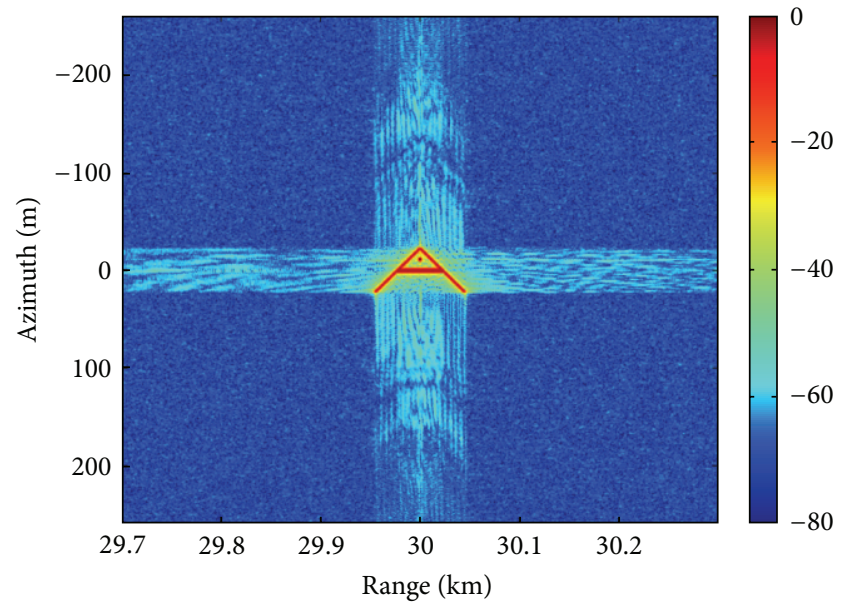

(b)

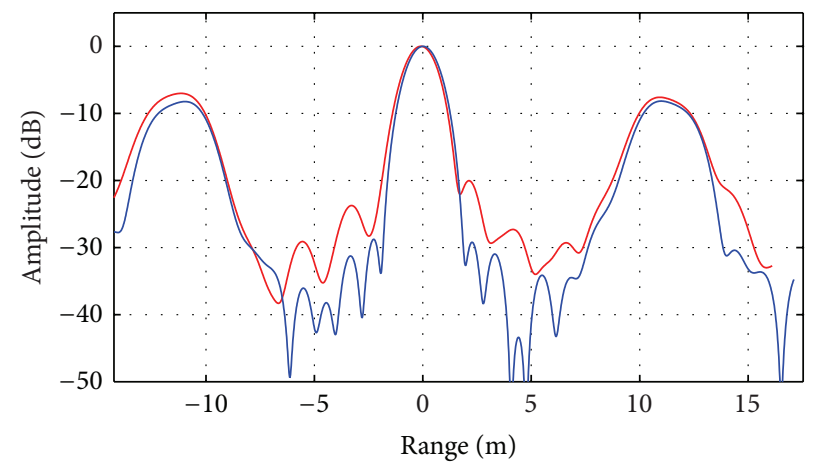

Without
With

(c)

FIGURE 7: Simulation results for OFDM-MIMO SAR. (a) Imaging result without correction of channel imbalance. (b) Imaging result with correction of channel imbalance. (c) Range comparison of the amplification of the point target in (a) and (b).

TABLE 2: Channel errors (CE).

\begin{tabular}{|c|c|c|c|c|c|c|}
\hline \multirow{2}{*}{ Channel $(m, n)$} & \multicolumn{2}{|c|}{ Exact CE } & \multicolumn{2}{|c|}{ Estimated CE for simulated data } & \multicolumn{2}{|c|}{ Estimated CE for constructed raw data } \\
\hline & $A_{k}$ & $\varphi_{k}\left({ }^{\circ}\right)$ & $\overline{A_{k}}$ & $\bar{\varphi}_{k}\left({ }^{\circ}\right)$ & $\overline{A_{k}}$ & $\bar{\varphi}_{k}\left({ }^{\circ}\right)$ \\
\hline 1,1 & 1 & 0 & 1 & 0 & 1 & 0 \\
\hline 1,2 & 1.3 & 25 & 1.296 & 24.308 & 1.253 & 28.201 \\
\hline 2,1 & 1.5 & 30 & 1.500 & 29.649 & 1.468 & 33.325 \\
\hline 2,2 & 1.4 & 45 & 1.401 & 44.426 & 1.352 & 42.720 \\
\hline
\end{tabular}




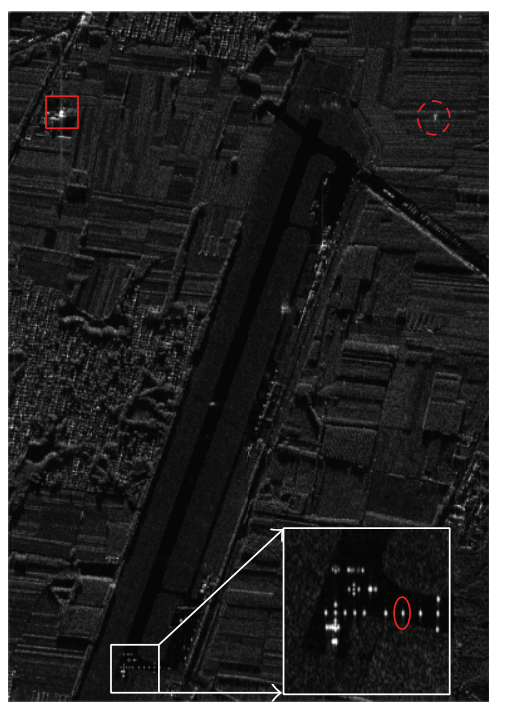

(a)

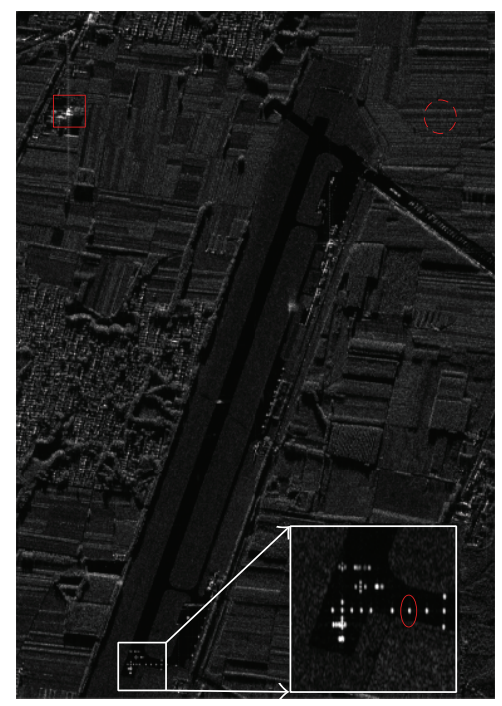

(b)

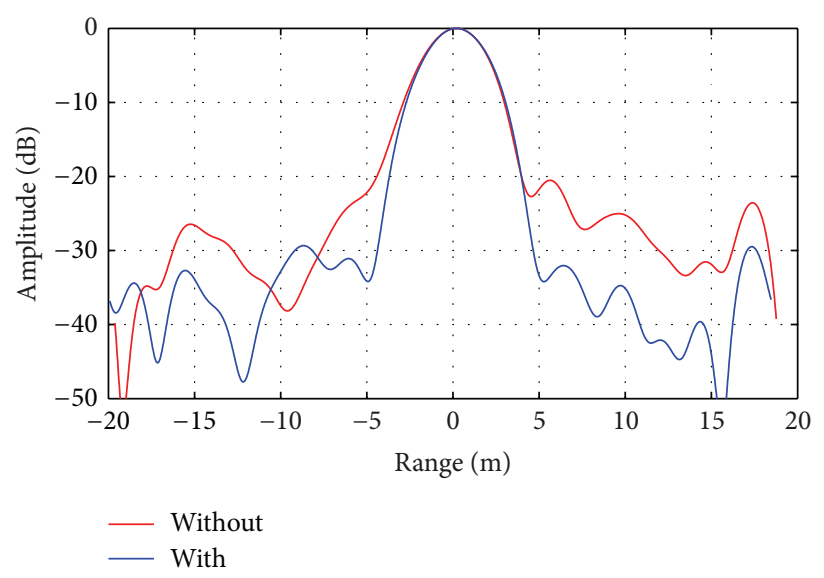

(c)

FIGURE 8: Real raw data results for OFDM-MIMO SAR. (a) Without correction. (b) With correction. (c) Range comparison of the amplification of the corner reflector.

with $f_{\eta}$ the Doppler frequency, $t_{1,1}=0, t_{1,2}=l_{a z} / 2 v_{s}=(3 / 4)$. (1/2PRF), $t_{2,1}=t_{1,2}-(1 / 4 \mathrm{PRF})$, and $t_{2,2}=t_{1,2}+t_{2,1}$. Then, the distribution of the EPCs for the four channels can be illustrated by the 2nd, 3rd, 4th, and 5th rows in Figure 5 where the dashed circles represent the discarded pulses to realize a two-time subsampling. With consideration of the channel errors specified in the 2nd and 3rd columns of Table 2, the four echo data of the MIMO system using STFCs are well constructed.

According to the error estimation process presented in Figure 6, the measured channel errors are listed in the 6th and 7 th columns of Table 2 . The final imaging results without and with correction of the channel imbalance are shown in Figures $8(a)$ and $8(b)$, respectively. The corner reflector used for external calibration is marked by ellipse in the two figures. After interpolation and amplification, the range comparison of the corner reflector in Figures 8(a) and 8(b) is shown in Figure $8(\mathrm{c})$, where one can see that the raising of the side lobes is eliminated by the compensation. Additionally, in
Figure 8(a) the peak amplitude of the ambiguity marked by dashed circle is $-15.89 \mathrm{~dB}$ while in Figure 8 (b) the ambiguity is not distinguishable from the background.

Therefore, for the constructed MIMO raw data, the presented correction method is also effective in removing the channel imbalance.

\section{Conclusion}

MIMO SAR using stepped frequency chirps is of great potential for future high-resolution wide-swath SAR missions. Channel imbalance is a key problem for this novel SAR mode, as it relates to the imaging performance. This paper proposed a simple and effective method to correct this channel imbalance, which was validated by simulation and real raw data experiments. Other problems, such as the system design and the orbit model, are not discussed in this paper. Nevertheless, they are also indispensable, especially when this novel mode is applied to spaceborne missions. 


\section{Conflict of Interests}

The authors declare that there is no conflict of interests regarding the publication of this paper.

\section{References}

[1] N. Gebert, G. Krieger, and M. A. Moreira, "Digital beamforming on receive: techniques and optimization strategies for highresolution wide-swath SAR imaging," IEEE Transactions on Aerospace and Electronic Systems, vol. 45, no. 2, pp. 564-592, 2009.

[2] W. Jing, M. Xing, C.-W. Qiu, Z. Bao, and T.-S. Yeo, "Unambiguous reconstruction and high-resolution imaging for multiplechannel SAR and Airborne experiment results," IEEE Geoscience and Remote Sensing Letters, vol. 6, no. 1, pp. 102-106, 2009.

[3] A. Currie and M. A. Brown, "Wide-swath SAR," IEE Proceedings, Part F: Radar and Signal Processing, vol. 139, no. 2, pp. 122135, 1992.

[4] W.-Q. Wang, "Space-time coding MIMO-OFDM SAR for highresolution imaging," IEEE Transactions on Geoscience and Remote Sensing, vol. 49, no. 8, pp. 3094-3104, 2011.

[5] Y. Song and R. Yang, "High resolution, wide swath SAR using sub-aperture sub-band technique," in Proceedings of the CIE International Conference on Radar (ICR '06), Shanghai, China, October 2006.

[6] G. Krieger, "MIMO-SAR: opportunities and pitfalls," IEEE Transactions on Geoscience and Remote Sensing, no. 99, pp. 118, 2013.

[7] J. H. G. Ender and J. Klare, "System architectures and algorithms for radar imaging by MIMO-SAR," in Proceedings of the IEEE Radar Conference (RADAR '09), Pasadena, Calif, USA, May 2009.

[8] A. W. Doerry, SAR Processing with Stepped Chirps and Phased Array Antennas, Sandia Nat. Lab., Albuquerque, NM, USA, 2006.

[9] P. Berens, "SAR with ultra-high range resolution using synthetic bandwidth," in Proceedings of the IEEE International Geoscience and Remote Sensing Symposium (IGARSS '99), pp. 1752-1754, Hamburg, Germany, July 1999.

[10] W. Nel, J. Tait, R. Lord, and A. Wilkinson, "The use of a frequency domain stepped frequency technique to obtain high range resolution on the CSIR X-Band SAR system," in Proceedings of the 6th IEEE AFRICON Conference in Africa, pp. 327-332, October 2002.

[11] J. H. G. Ender and A. R. Brenner, "PAMIR—a wideband phased array SAR/MTI system," IEE Proceedings: Radar, Sonar and Navigation, vol. 150, no. 3, pp. 165-172, 2003.

[12] C.-G. Gao, Y.-K. Deng, and J. Feng, "Theoretical analysis on the mismatch influence of displaced phase center multiple-beam SAR systems," Journal of Electronics \& Information Technology, vol. 33, no. 8, pp. 1828-1832, 2011.

[13] X. Luo, R. Wang, Y. Deng, and W. Xu, "Influences of channel errors and interference on the OFDM-MIMO SAR," in Proceedings of the IEEE Radar Conference, pp. 1-5, Ottawa, Canada, 2013.

[14] Y. Deng, H. Zheng, R. Wang, J. Feng, and Y. Liu, "Internal calibration for stepped-frequency chirp SAR imaging," IEEE Geoscience and Remote Sensing Letters, vol. 8, no. 6, pp. 11051109, 2011.
[15] J. Feng, C. Gao, Y. Zhang, and R. Wang, "Phase mismatch calibration of the multichannel SAR based on azimuth crosscorrelation," IEEE Geoscience and Remote Sensing Letters, vol. 10, no. 4, pp. 903-907, 2013.

[16] L. Zhang, M.-D. Xing, C.-W. Qiu, and Z. Bao, "Adaptive two-step calibration for high-resolution and wide-swath SAR imaging," IET Radar, Sonar and Navigation, vol. 4, no. 4, pp. 548-559, 2010.

[17] J. C. Curlander and R. N. Mcdonough, Synthetic Aperture Radar: Systems and Signal Processing, John Wiley \& Sons, New York, NY, USA, 1991.

[18] I. G. Cumming and F. H. Wong, Digital Processing of Synthetic Aperture Radar Data: Algorithms and Implementation, Artech House, Norwood, Mass, USA, 2005. 

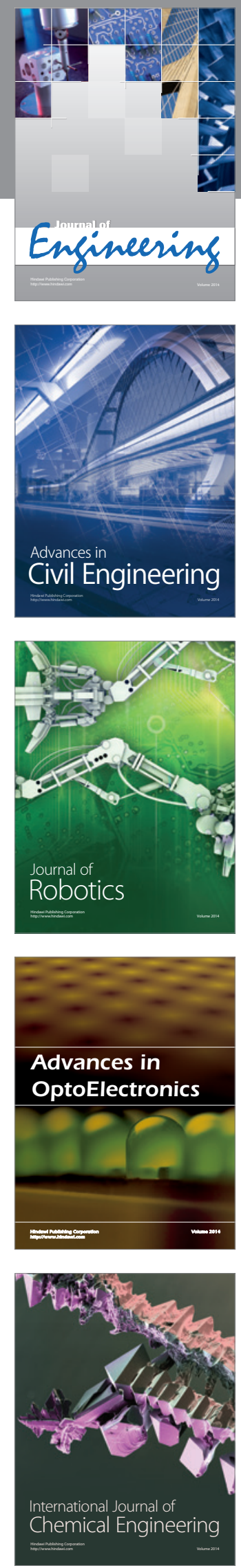

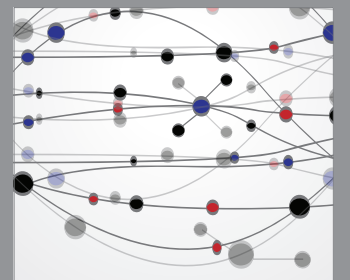

The Scientific World Journal
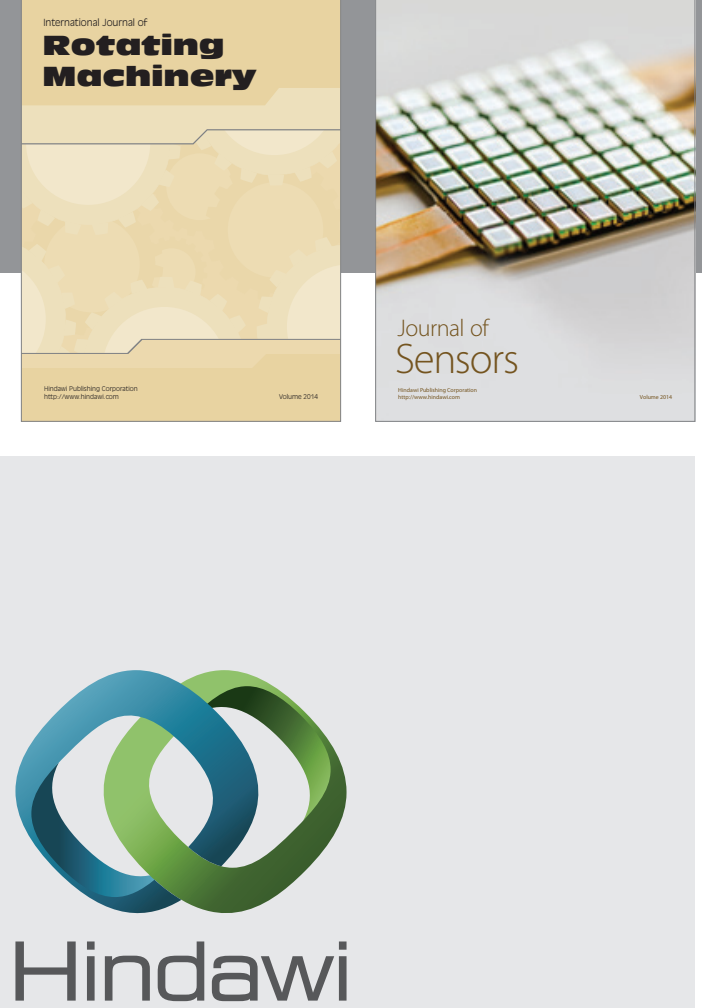

Submit your manuscripts at http://www.hindawi.com
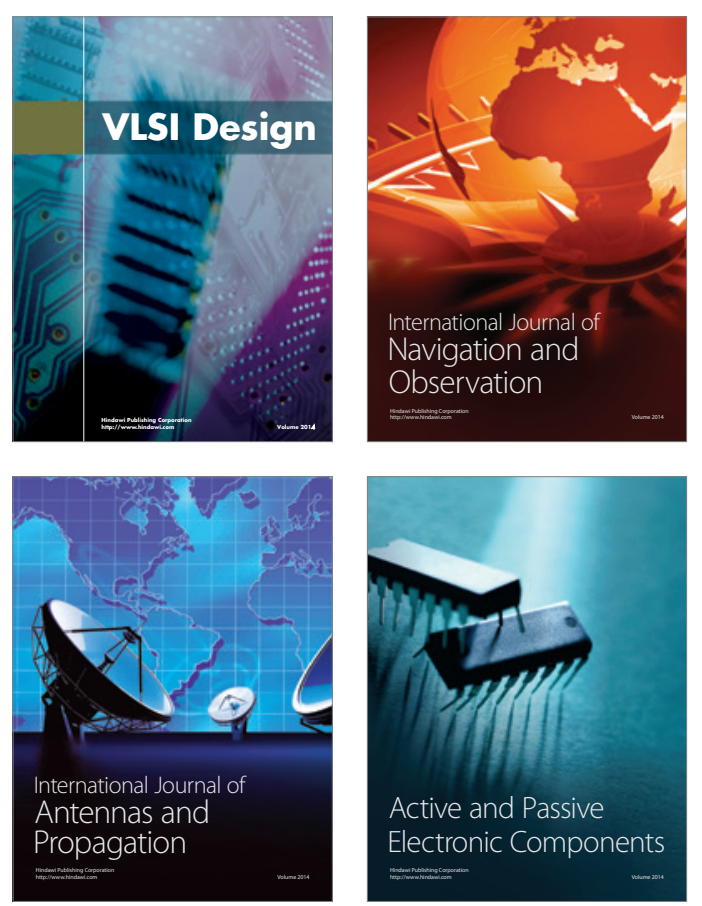
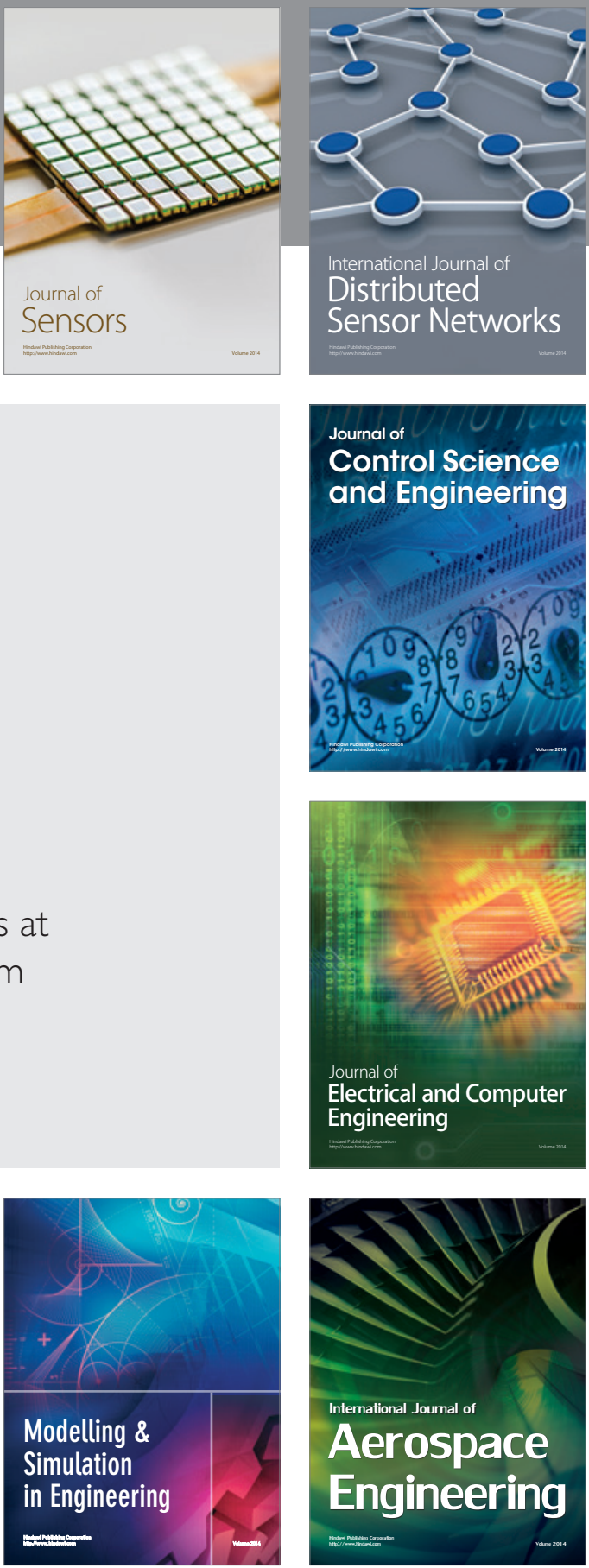

Journal of

Control Science

and Engineering
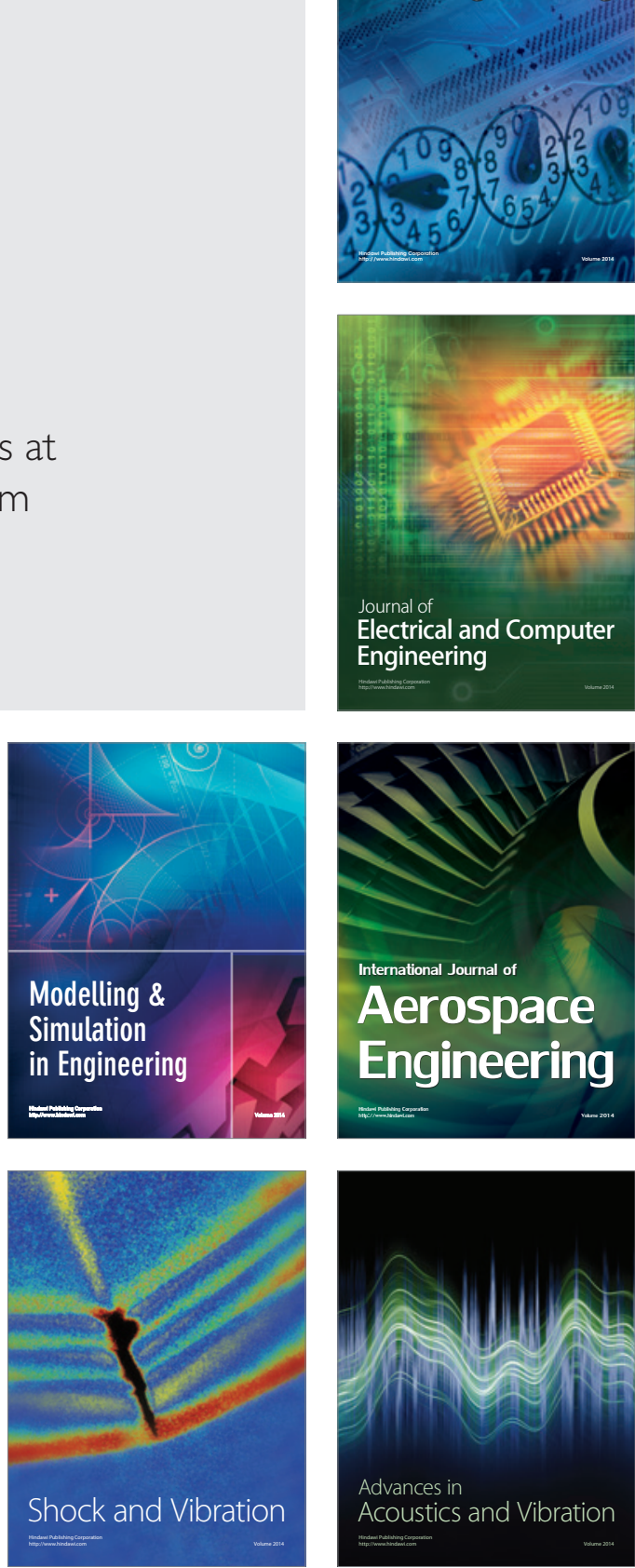